\title{
Dynamic Movement Primitives for Cooperative Manipulation and Synchronized Motions
}

\author{
Jonas Umlauft, Dominik Sieber and Sandra Hirche
}

\begin{abstract}
Cooperative manipulation, where several robots jointly manipulate an object from an initial configuration to a final configuration while preserving the robot formation, poses a great challenge in robotics. Here, we treat the problem of designing motion primitives for cooperative manipulation such that the robots move in formation and are robust with respect to external disturbances. Individual robot trajectories are generated by Dynamic Movement Primitives (DMPs) and coupled by a formation control approach enabling the DMPtrajectories to preserve a given formation while performing the manipulation. The proposed control scheme achieves an increased adaptability under external disturbances. The approach is evaluated in a full-scale experiment with two prototypical cooperative manipulation and synchronized motion tasks.
\end{abstract}

\section{INTRODUCTION}

Physical cooperation of robotic manipulators is important in a wide variety of tasks such as joint object manipulation under formation constraints and synchronized handover tasks. Application domains are for example in manufacturing, logistics, disaster relief, and service robotics. A cooperative manipulation task is considered effectively accomplished when the multi-robot team jointly manipulates the object from an initial configuration to a final configuration without damaging the object, i.e. without exerting excessive forces. If the robots are able to maintain a rigid formation, , the internal forces are significantly reduced [1]. In order to achieve successful task execution, a flexible, robust, and distributed control mechanism is required. The control architecture is called distributed if there is no central coordinating entity, i.e. each manipulator has its own local controller which may communicate with other local controllers to achieve the overall control goal.

Different control approaches have been proposed for cooperative manipulation. A popular approach is based on distributed impedance control, see for example [2]-[4]. Those approaches decouple the path planning and the control, i.e. individual trajectories are planned for each robotic manipulator taking into account the object geometry and grasp points [5], [6]. If feedback is missing in the path planning, those approaches are very sensitive to the initial configuration, the model accuracy, and in particular to external disturbances from the environment [7]. Embedding the formation rigidity constraint in an LQR-like optimal control problem is proposed in [8]. However, the approach only considers the maintenance of the rigidity constraint

\footnotetext{
All authors are with the Institute for Information-Oriented Control, Department of Electrical Engineering and Information Technology, Technische Universität München, D-80290 Munich, Germany. \{jonas.umlauft , dominik.sieber, hirche\}atum.de
}

and is not suitable for establishing formations and synchronized movements. A powerful tool for representing discrete and periodic trajectories are Dynamic Movement Primitives (DMPs) developed in [9] and [10]. DMPs are mainly used for imitation and learning tasks of a single manipulator and are therefore useful for representing and generating human-like movements. To the best of the authors knowledge, multiple dynamic motor primitives have not yet been investigated in depth for cooperative manipulation except for preliminary work in [11], where a bimanual task with force feedback is considered without considering more than two DMPs.

The main contribution of the paper is a novel control approach for cooperative manipulation and movement synchronization. The approach is based on formation control and coupled DMP-based trajectory planning. Here, DMPs generate individual manipulator trajectories to a desired final configuration, which is in agreement with the initial configuration of the multi-robot team. During the tracking phase, it may happen that individual DMPs violate the formation rigidity or disturbances occur on a single manipulator. The issue is circumvented in our approach by using a formation-preserving feedback for each DMP. Minor remaining deviations from the formation are accommodated by local impedance control laws in each manipulator such that they react compliantly to their environment. Our proposed control and trajectory generation approach is evaluated in experiments and its robustness against perturbations and reduced internal forces on the object are demonstrated for cooperative manipulation and synchronized motion tasks.

The remainder of the paper is structured as follows. Section II formally describes DMPs and formation control. The proposed extension for DMPs for cooperative manipulation is presented in Section III, the experimental evaluation is presented in Section IV.

Notation: Bold symbols denote vectors. $\|\boldsymbol{a}\|$ represents the Euclidian norm of vector $\boldsymbol{a} . \boldsymbol{a}_{i}$ relates the variable to $i$ th manipulator. $\boldsymbol{a}_{i, k}$ relates the variable to the $k$ th state of manipulator $i$. The $n \times n$ identity matrix is denoted as $I_{n}$.

\section{BACKGROUND}

In this section we present the necessary background on DMPs, formation control, and impedance control.

\section{A. Dynamic Movement Primitives (DMPs)}

DMPs provide generalized trajectories for discrete and periodic movements of a robotic manipulator to drive from an initial position $x_{0}$ to a goal position $g$. DMPs can represent complex movements while being capable to incorporate 
feedback in real-time [12]. The following attractor dynamic ensures that the solution of the point attractive system, the trajectory $x(t)$, converges towards the goal $g$ [13]

$$
\begin{aligned}
& \tau \dot{z}=\alpha(\beta(g-x)-z)+f(s) \\
& \tau \dot{x}=z
\end{aligned}
$$

with the nonlinearity $f(s)$ defined as

$$
f(s)=\frac{\sum_{i=1}^{W} w_{i} \Psi_{i}(s)}{\sum_{i=1}^{W} \Psi_{i}(s)} s, \Psi_{i}(s)=\exp \left(-h_{i}\left(s-b_{i}\right)^{2}\right)
$$

where $w_{i}, b_{i}, h_{i}$ are the weights, the centers and the radii, respectively, of the radial basis function distributed along the trajectory. Those parameters are determined during the learning process using Locally Weighted Projection Regression (LWPR) [14]. The parameters $\alpha, \beta>0$ are set prior to the learning process. The timing parameter $\tau>0$ is adjusted before the execution of the movement to speed it up or slow it down compared to the demonstration. A phase variable $s$ is used in (3) instead of time to make the dependency of $f(s)$ on time more implicit [13]. The dynamics of the phase variable $s$ are defined by a canonical system

$$
\tau \dot{s}=-\gamma s, \quad \gamma>0 .
$$

The advantage of using the phase variable $s$ instead of explicit time is that we are able to modify the evolution of time by appropriately adapting (4) during execution [13]. Thus by decelerating the canonical system, it is possible to slow down the execution of the steering function $f(s)$, which reduces the speed of the movement, e.g. in case of disturbances. Obviously, $s \rightarrow 0$ as $t \rightarrow \infty$ and therefore the nonlinear function $f(s)$ tends to zero as time increases. Thus, the asymptotically stable equilibrium point of the point attractive system (1),(2) is

$$
\left[\begin{array}{ll}
z^{*} & x^{*}
\end{array}\right]=\left[\begin{array}{ll}
0 & g
\end{array}\right] .
$$

As a result trajectories $x(t)$ generated by DMPs ensure the discrete movement from the initial point $x_{0}$ to the goal $g$. DMPs are also used to generate trajectories for periodic movements. In contrast to discrete movements the steering function $f$ will not approach zero because it is a weighted sum of periodic functions $\Gamma(\phi)$ multiplied by the amplitude $r$ of the oscillation.

$$
\begin{aligned}
f(\phi) & =\frac{\sum_{i=1}^{W} w_{i} \Gamma_{i}(\phi)}{\sum_{i=1}^{W} \Gamma_{i}(\phi)} r \\
\Gamma(\phi) & =\exp \left(-h_{i}\left(\cos \left(\phi-b_{i}\right)-1\right)\right),
\end{aligned}
$$

The function $f(\phi)$ is used to modify the dynamics of the basic second-order system (1), (2) [15]. Rewriting (1) and (2) using the frequency of the oscillation $\Omega=1 / \tau$ yields

$$
\begin{aligned}
& \dot{z}=\Omega(\alpha(\beta(g-x)-z)+f(\phi)) \\
& \dot{x}=\Omega z .
\end{aligned}
$$

For periodical movements, the phase variable $\phi$ increases constantly as opposed to $s$ and is given by

$$
\dot{\phi}=\Omega \text {. }
$$

\section{B. Formation Control}

A common approach to formation control is to use a Artificial Potential Field (APF) in order to achieve a desired distance $d_{i j}$ between two agents $i$ and $j$ [16]. Often a quadratic potential field $V_{i j}\left(\left\|\boldsymbol{x}_{i}-\boldsymbol{x}_{j}\right\|\right)$ is considered which has its global minimum at the desired distance $d_{i j}$

$$
V_{i j}\left(\left\|\boldsymbol{x}_{i}-\boldsymbol{x}_{j}\right\|\right)=\frac{1}{2}\left(\left\|\boldsymbol{x}_{i}-\boldsymbol{x}_{j}\right\|-d_{i j}\right)^{2} .
$$

From the potential field, a control signal $\boldsymbol{u}_{i}$ for the $i$-th agent is derived based on the gradient descent

$$
\begin{aligned}
\boldsymbol{u}_{i} & =-\sum_{j \in N_{i}} \delta_{i j} \frac{\partial V_{i j}\left(\left\|\boldsymbol{x}_{i}-\boldsymbol{x}_{j}\right\|\right)}{\partial \boldsymbol{x}_{i}} \\
& =-\sum_{j \in N_{i}} \delta_{i j} \frac{\boldsymbol{x}_{i}-\boldsymbol{x}_{j}}{\left\|\boldsymbol{x}_{i}-\boldsymbol{x}_{j}\right\|}\left(\left\|\boldsymbol{x}_{i}-\boldsymbol{x}_{j}\right\|-d_{i j}\right),
\end{aligned}
$$

where $\delta_{i j}$ is a positive parameter that is used to adjust the speed of convergence to the desired formation. Under this control law all agents converge to the desired formation [17].

\section{Impedance Control}

In order to accommodate for minor deviations of the desired position trajectories from the formation constraints we propose to employ impedance control here. Therefore, the manipulator measures contact forces at its end-effector. The Cartesian impedance control law is described as following

$$
M \ddot{e}+D \dot{e}+K e=\zeta,
$$

where $M, D, K \in \mathbb{R}^{n \times n}$ are the mass, the damping and the stiffness matrices, respectively. Here, $n$ denotes the workspace dimension, $\zeta \in \mathbb{R}^{n}$ is the applied force and $\boldsymbol{e}=\overline{\boldsymbol{x}}-\boldsymbol{x}$ is the deviation of the actual end effector position $\overline{\boldsymbol{x}} \in \mathbb{R}^{n}$ from the desired position $\boldsymbol{x} \in \mathbb{R}^{n}$. The trajectories for the desired positions $\boldsymbol{x}$, velocities $\dot{\boldsymbol{x}}$, and accelerations $\ddot{\boldsymbol{x}}$ are required as input to the impedance control law and are generated using DMPs. The position $\boldsymbol{x}_{i}$ is expressed in a world coordinate system $\Sigma_{w}$ which requires that each endeffector is aware of its transformation to a common reference.

\section{CoOperative DMPs}

The goal is to introduce a novel approach for DMP-based formation control for generating cooperative movements of $N$ manipulators in a $n$-dimensional workspace. For the proposed control law, rotational motions of each manipulator are neglected so that the DMPs are represented by decoupled dynamics for each Cartesian degree of freedom (DoF).

For each degree of freedom we use a single point attractive system (1), (2) and one canonical system (4). Here, $\boldsymbol{x}_{i}$ denotes the state of the manipulator $i$, which consists of the Cartesian positions $x_{i, k}$ in each dimension $k$ of the workspace, thus $\boldsymbol{x}_{i}=\left[x_{i, 1}, \ldots, x_{i, n}\right]^{T}$. The states of all manipulators are concatenated into $\boldsymbol{x}=\left[\boldsymbol{x}_{1}^{T}, \ldots, \boldsymbol{x}_{N}^{T}\right]^{T}$. Note that, $x_{i, k}$ describes the single state of a manipulator corresponding to the $x$ used in (1),(2). The goal configuration $\boldsymbol{g}$ is an accordingly concatenated vector. The desired trajectory $\boldsymbol{x}, \dot{\boldsymbol{x}}$ which is generated by $n \times N$ DMPs and 
tracked by the impedance control law (11) requires to satisfy the rigidity constraint among the cooperating manipulators. Since each DMP only knows its own state, we establish a communication link between neighboring DMPs and employ a cooperative term to modify the individual states. A DMP is called a neighbor of another DMP, if formation rigidity requires a desired distance to be maintained. Here, each DMP relies on the relative position to its neighbors and is attractive to the desired formation even under external disturbances. The graph representing the communication structure among the systems of DMPs which is formulated by the neighboring set is chosen such that the minimum number of neighbors for rigidity of the formation is guaranteed. For more details on formation rigidity the reader is referred to [18].

In order to extend DMPs for cooperative manipulation the ability to react to the movements of other manipulators is important. Therefore we introduce a cooperation feedback term into the DMPs. Our approach is inspired by [9] where DMPs are enhanced to react to external perturbations. According to [12] the point attractive system (1) and (2) can be augmented to incorporate feedback as follows

$$
\begin{aligned}
\tau \dot{z} & =\alpha(\beta(g-x)-z)+f(s) \\
\tau \dot{x} & =z+\alpha_{\text {err }}(\tilde{x}-x),
\end{aligned}
$$

where $\tilde{x}$ denotes the measured position of the manipulator, whereas $x$ is the desired position. The slowing of the phase variable $s$ until the error is reduced is a key feature of this approach. Note that the phase variable $s$ directly controls the steering function $f(s)$, which also decelerates proceeding until the error is compensated. According to [15] the canonical system is augmented to

$$
\tau \dot{s}=-\frac{\gamma s}{1+\gamma_{\mathrm{err}}(\tilde{x}-x)^{2}} .
$$

Here, we propose to use a feedback term to enforce the formation based on the input given by the formation control law in (10). Therefore, a deviation in the relative distance of the neighbors to the desired distance $d_{i j}$ is interpreted as an error term and is compensated before further proceeding to the goal. The point attractive system and the canonical system of manipulator $i$ in dimension $k$ is reformulated as

$$
\begin{aligned}
\tau \dot{z}_{i, k} & =\alpha_{i, k}\left(\beta_{i, k}\left(g_{i, k}-x_{i, k}\right)-z_{i, k}\right)+f_{i, k}\left(s_{i, k}\right), \\
\tau \dot{x}_{i, k} & =z_{i, k}+\kappa_{i, k} c_{i, k}(\boldsymbol{x}), \\
\tau \dot{s}_{i, k} & =-\frac{\gamma_{i, k} s_{i, k}}{1+\eta_{i, k} c_{i, k}^{2}(\boldsymbol{x})} .
\end{aligned}
$$

where $\eta_{i, k}$ and $\kappa_{i, k}$ are both positive weighting parameters for the cooperation term $c_{i, k}(\boldsymbol{x})$ which is the $k$-th dimension of $\boldsymbol{c}_{i}(\boldsymbol{x})$. For the cooperation term we use the input of the control law for formation control $\boldsymbol{c}_{i}(x)=\boldsymbol{u}_{i}$, i.e.

$$
\boldsymbol{c}_{i}(\boldsymbol{x})=-\sum_{j \in N_{i}} \delta_{i j} \frac{\boldsymbol{x}_{i}-\boldsymbol{x}_{j}}{\left\|\boldsymbol{x}_{i}-\boldsymbol{x}_{j}\right\|}\left(\left\|\boldsymbol{x}_{i}-\boldsymbol{x}_{j}\right\|-d_{i j}\right) \text {. }
$$

Thus, the cooperation term is zero if the desired distances to all neighbors are maintained and (15), (16), (17) are equivalent to the original DMPs from (1), (2) and (4),

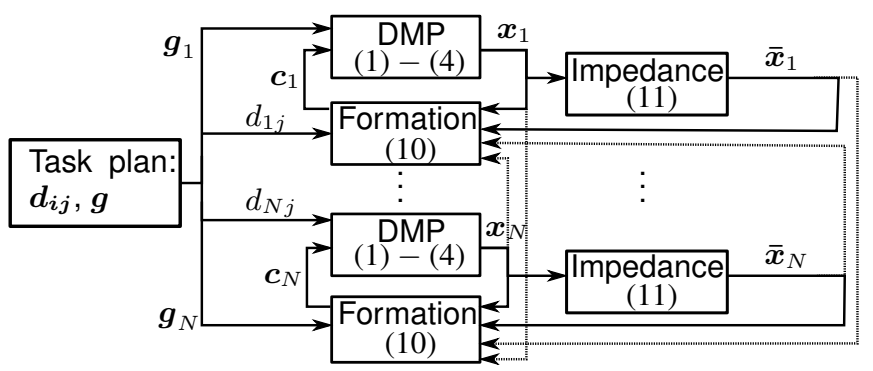

Fig. 1: General control approach for cooperative DMP

respectively. In case $\boldsymbol{c}_{i}(\boldsymbol{x})$ increases the canonical system is decelerated and the steering function is delayed. Through $\boldsymbol{c}_{i}(\boldsymbol{x}) \neq \mathbf{0}$, an additional acceleration on the point attractive system is acting, which tends to restore the desired distance.

Choosing the parameters $\eta_{i, k}$ and $\kappa_{i, k}$ such that $\eta_{i, k}, \kappa_{i, k} \gg 1 \forall i, k$, a better formation maintenance is ensured but it might take more time to reach the goal. In case the formation is violated, higher $\eta_{i, k}$ decelerate the canonical systems and thus the execution of the steering functions. Increasing $\kappa_{i, k}$ affects the point attractive systems to react faster to disturbances of the formation. Given this enhancement, DMPs are applicable for a cooperative manipulation task or the generation of synchronized periodic movement. The schematic overview of the control scheme and its references in this work are illustrated in Fig. 1. A task plan provides the goal configurations $g$ and the inter-robot distances $d_{i j}$. The individual desired trajectories are generated by DMPs and then tracked by the impedance. A cooperative feedback term is introduced to enforce the desired distance by a formation control law.

\section{Equilibria and Domain of Attraction}

As the point attracting system (15) and (16) and the canonical system (17) are modified we investigate the equilibria of the attractor landscape of the augmented system. Analogously to the argumentation in [9], the stability properties of the canonical system are preserved, i.e. the equilibrium $s_{i, k}^{*}=0$ of (17) is asymptotically stable and derived from

$$
\tau \dot{s}_{i, k}^{*}=-\frac{\gamma_{i, k} s_{i, k}^{*}}{1+\eta_{i, k} c_{i, k}^{2}\left(\boldsymbol{x}^{*}\right)} \stackrel{!}{=} 0 \rightarrow s_{i, k}^{*}=0 \forall i, k .
$$

Recalling the definition of the nonlinear function $f(s)$ in (3), it is obvious that $f(s) \rightarrow 0$ for $s \rightarrow 0$. Therefore the equilibrium point of system (15), (16) is derived using

$$
\begin{aligned}
& \tau \dot{z}_{i, k}^{*}=\alpha_{i, k}\left(\beta_{i, k}\left(g_{i, k}-x_{i, k}^{*}\right)-z_{i, k}^{*}\right) \stackrel{!}{=} 0 \\
& \tau \dot{x}_{i, k}^{*}=z_{i, k}^{*}+\kappa_{i, k} c_{i, k}\left(\boldsymbol{x}^{*}\right) \stackrel{!}{=} 0 .
\end{aligned}
$$

From (19) one concludes that $z_{i, k}^{*}=-\kappa_{i, k} c_{i, k}\left(\boldsymbol{x}^{*}\right)$. Using this in (18) we obtain the following condition for equilibria

$$
x_{i, k}^{*}=g_{i, k}+\frac{\kappa_{i, k}}{\beta_{i, k}} c_{i, k}\left(\boldsymbol{x}^{*}\right) \quad \forall i, k
$$

For illustration we compute the equilibria for two agents. The computation of the equilibria for $N$ agents from (20) is straightforward. For simplicity of the subsequent analysis we set $\beta_{i, k}=\kappa_{i, k}=1$ and $\delta_{i j}=1, \forall i, j$. Using $d_{12}=d_{21}=\left\|g_{1}-g_{2}\right\|$, (20) results in $x_{1}^{*}=g_{1}-\left(x_{1}^{*}-\right.$ 


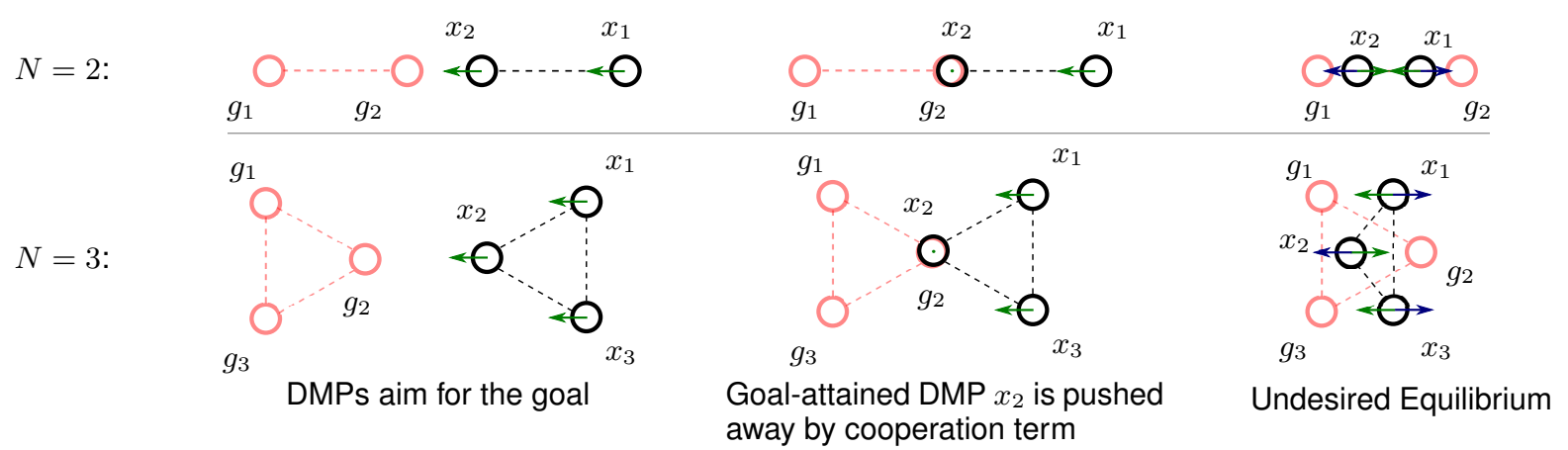

Fig. 2: Top: Two manipulators $x_{1}, x_{2}$ approach the goal configurations $g_{1}, g_{2}$ along the domain of attraction in opposite sequence to the goals. In the middle column, manipulator $x_{2}$ reaches its goal position $g_{2}$ first, but $x_{1}$ endeavors to reach goal $g_{1}$ which is $2 d_{12}$ away. Due to the virtual coupling force of the cooperation term, $x_{1}$ gets pushed away until both manipulators reach the undesired equilibrium in balance. Bottom: One undesired equilibrium for three manipulators .

$\left.x_{2}^{*}\right)\left(1-\frac{\left\|g_{1}-g_{2}\right\|}{\left\|x_{1}^{*}-x_{2}^{*}\right\|}\right)$ and $x_{2}^{*}=g_{2}-\left(x_{2}^{*}-x_{1}^{*}\right)\left(1-\frac{\left\|g_{1}-g_{2}\right\|}{\left\|x_{2}^{*}-x_{1}^{*}\right\|}\right)$. As a result we obtain the desired equilibrium

$$
\boldsymbol{x}_{1}^{*}=\boldsymbol{g}_{1}, \quad \boldsymbol{x}_{2}^{*}=\boldsymbol{g}_{2}
$$

and an undesired equilibrium

$$
\boldsymbol{x}_{1}^{*}=\boldsymbol{g}_{2}+\frac{1}{3}\left(\boldsymbol{g}_{1}-\boldsymbol{g}_{2}\right), \quad \boldsymbol{x}_{2}^{*}=\boldsymbol{g}_{2}+\frac{2}{3}\left(\boldsymbol{g}_{1}-\boldsymbol{g}_{2}\right) .
$$

In the desired equilibrium the agents have attained their goal positions $g_{1}$ and $g_{2}$ which are formation consistent. In the undesired equilibrium the agents do not reach their goal positions and do not maintain the formation. The undesired equilibrium is approached if the two vector fields of goal attraction $x_{i, k}^{*}-g_{i, k}$ and cooperation term $\frac{\kappa_{i, k}}{\beta_{i, k}} c_{i, k}\left(\boldsymbol{x}^{*}\right)$ cancel each other out, which is illustrated in the top row of Fig. 2. A cancellation is possible if the vectors are linearly dependent and point in opposite directions. This explanation also provides an intuitive understanding of the domain of attraction of the undesired equilibrium. The domain of attraction describes the subspace, from which the agents start and can end up in an undesired equilibrium. Here, the domain of attraction of the undesired equilibrium is given by a line through the goal configurations with both agents being in switched configuration (compared to the goal positions). For practical application the undesired equilibrium is largely irrelevant as small perturbations allow the agents to escape this domain. For $N=3$ one undesired equilibrium is illustrated in the bottom row of Fig. 2. In case of more agents the approach is similar, however the undesired equilibria and their domain of attraction is more complicated to compute; its formal analysis is still part of ongoing research.

\section{Experimental Evaluation}

\section{A. Experimental Setup}

The experimental setup consists of two commercially available KUKA LWR 4+ (light-weight robot), see Fig. 3. Both manipulators are assembled on top of the same mobile platform in order to avoid issues such as communication and kinematic uncertainties at this preliminary stage. A Cartesian impedance control scheme (11) is employed to ensure compliance of the end-effectors. Both Cartesian positions are captured by a QualiSys motion capture system. A workspace extension by a mobile platform of both robotic manipulators is accomplished according to the approach presented in [3]. The manipulator motion and the mobile platform motion are kinematically decoupled in task-space with an employed potential function. The parameters for DMPs are chosen equally for each manipulator in all dimensions as exhibited in Table I distinguished in discrete and periodic movements.

\begin{tabular}{llcc}
\hline & Eq. & $\begin{array}{c}\text { Discrete } \\
\text { Movement }\end{array}$ & $\begin{array}{c}\text { Periodic } \\
\text { movement }\end{array}$ \\
\hline Attractor system & $(15)$ & $\alpha_{i, k}=5, \beta_{i, k}=0.01, \forall i, k$ \\
Canonical system & $(17)$ & $\gamma_{i, k}=0.01, \forall i, k$ \\
Time scaling & $(16)$ & $\tau=20 \frac{1}{s}$ & $\Omega=1 s$ \\
$\begin{array}{l}\text { Cooperation weight } \\
\text { with cooperation }\end{array}$ & $(16),(17)$ & $\eta_{i, k}=\kappa_{i, k}=10$ & $\eta_{i, k}=\kappa_{i, k}=1$ \\
$\begin{array}{l}\text { Cooperation weight } \\
\text { without cooperation }\end{array}$ & $(16),(17)$ & $\eta_{i, k}=\kappa_{i, k}=0, \forall i, k$ \\
Impedance parame- & $(11)$ & $M=10 I_{3}, D=120 I_{3}, K=160 I_{3}$
\end{tabular}

TABLE I: Control parameters used in experiments

\section{B. Discrete Movement in Cooperative Manipulation}

The objective of this first task is to demonstrate that cooperating DMPs maintain the formation in case of disturbances and compensate the deviations caused by other

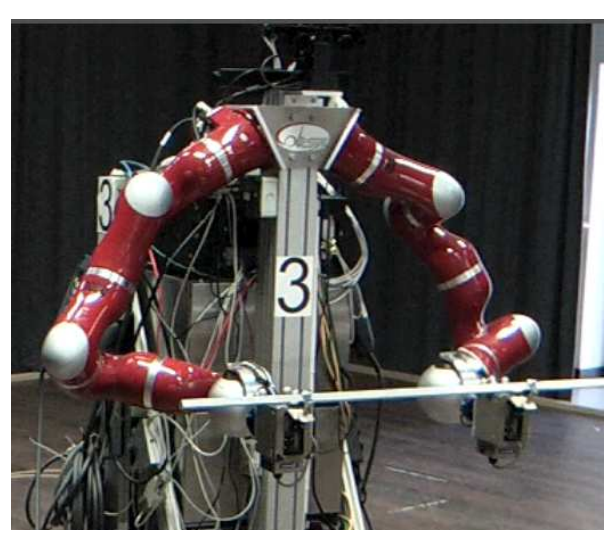

Fig. 3: The robot platform used in the experiments 

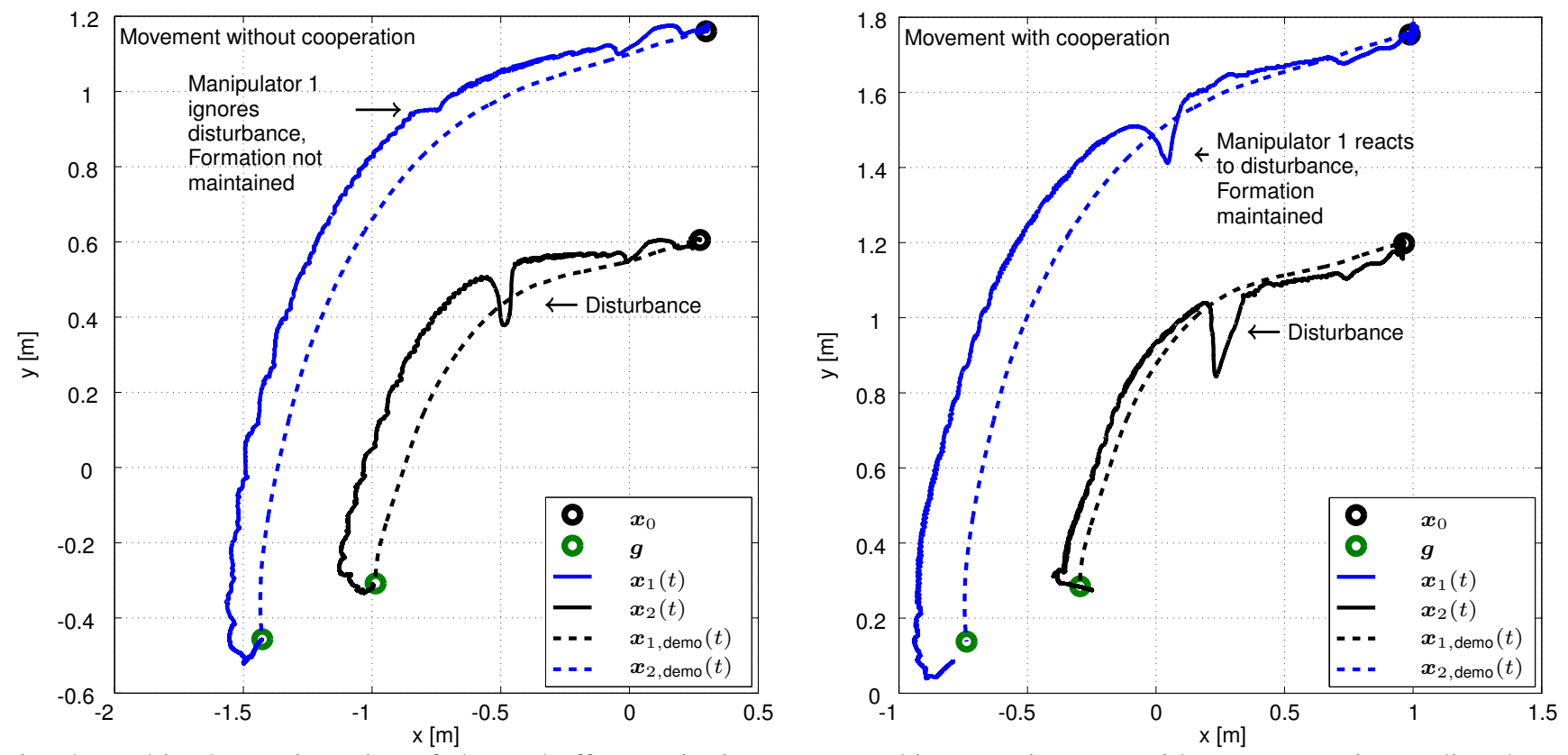

Fig. 4: Multi-robot trajectories of the end-effectors in 2D space tracking a trajectory. Without cooperation a disturbance acting on one manipulator has no effect on the other. In cooperation both manipulators are virtually coupled.

robotic manipulators. For this experiment, the robot performs a planar cooperative manipulation task with two endeffectors. The desired trajectory $\boldsymbol{x}_{\mathrm{demo}}$ is generated from demonstrations by LWPR [14]. The task is to carry a beam in a discrete movement. In order to demonstrate the efficacy of our proposed approach, we remove the beam between the manipulators and identify the effect of cooperation without physical coupling. During the movement a disturbance is imposed on one robotic manipulator by pushing against it. Due to the impedance control law the manipulator deviates from the desired trajectory $\boldsymbol{x}_{2}$.

The results of this experiment are shown in Fig. 4. It is obvious that a disturbance acting on manipulator has no effect on the other one if cooperation feedback is turned off. When the cooperation feedback is turned on, the disturbance affects both manipulators simultaneously. As desired, the formation is restored by the cooperative DMP approach.

\section{Motion Synchronization}

The second experiment investigates the idea of cooperating DMPs for periodic movements. For this experiment both manipulators oscillate in phase with the same frequency and amplitude in one direction. During this experiment we turn the formation feedback on and off and observe the synchronization of two manipulators after being out of phase. We simulate a disturbance on one of the manipulators by fixing it manually for a short time. With this experiments, we demonstrate that disturbances acting on one of the manipulator also affect the trajectories of the other manipulator.

The results of this experiment with and without cooperation are shown in Fig. 5. After one cycle $(\approx 8 s)$ the cooperation is turned off and the two manipulators are drifting apart. At $t \approx 18 \mathrm{~s}$ manipulator 1 is fixed at its current position manually, simulating a disturbance. After it is released at $t \approx 22 s$ it keeps oscillating but the two manipulators are not in phase. So at $t \approx 26 \mathrm{~s}$ the cooperation is turned on again and a both manipulators are synchronizing.

\section{Reducing internal forces}

In a further experiment we show the reduction of internal forces acting on the object. In this experiment both manipulators follow a discrete movement. A beam is rotationally free fixed at the manipulators. For the discrete movement individual trajectories are generated using DMPs which violate the formation constraint.

Using DMPs without cooperation results in significant internal forces acting on the object because both manipulators follow trajectories that deviate from the desired formation. Due to impedance control the deviation causes a force on the object defined by (11). Using cooperative DMPs the trajectories are adjusted such that they match the desired formation. As desired, this results in reduction of forces acting on the object as shown in Fig. 6.

\section{CONCLUSIONS}

In this paper we propose a novel control approach for cooperative manipulation and synchronization based on cooperative dynamic movement primitives (DMPs). A cooperation feedback term based on an artificial potential field for formation control is introduced into the original DMP formulation. The equilibria analysis provides desired and undesired equilibria, however, the undesired equilibria turn out to be of no practical relevance. The effectiveness and quality of the proposed controller with respect to disturbances is successfully demonstrated in experiments.

\section{ACKNOWLEDGMENTS}

The work is partly supported by the German Research Foundation (DFG) excellence initiative research cluster "Cognition for 


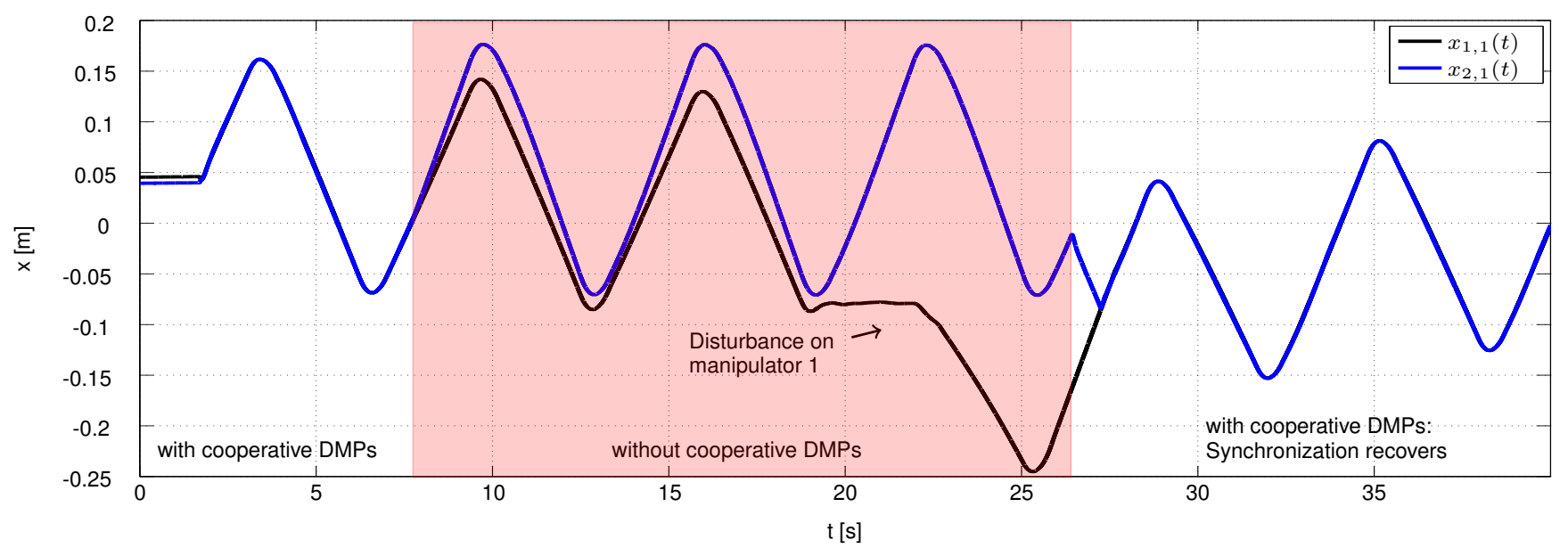

Fig. 5: Synchronization of two manipulators performing a periodic movement

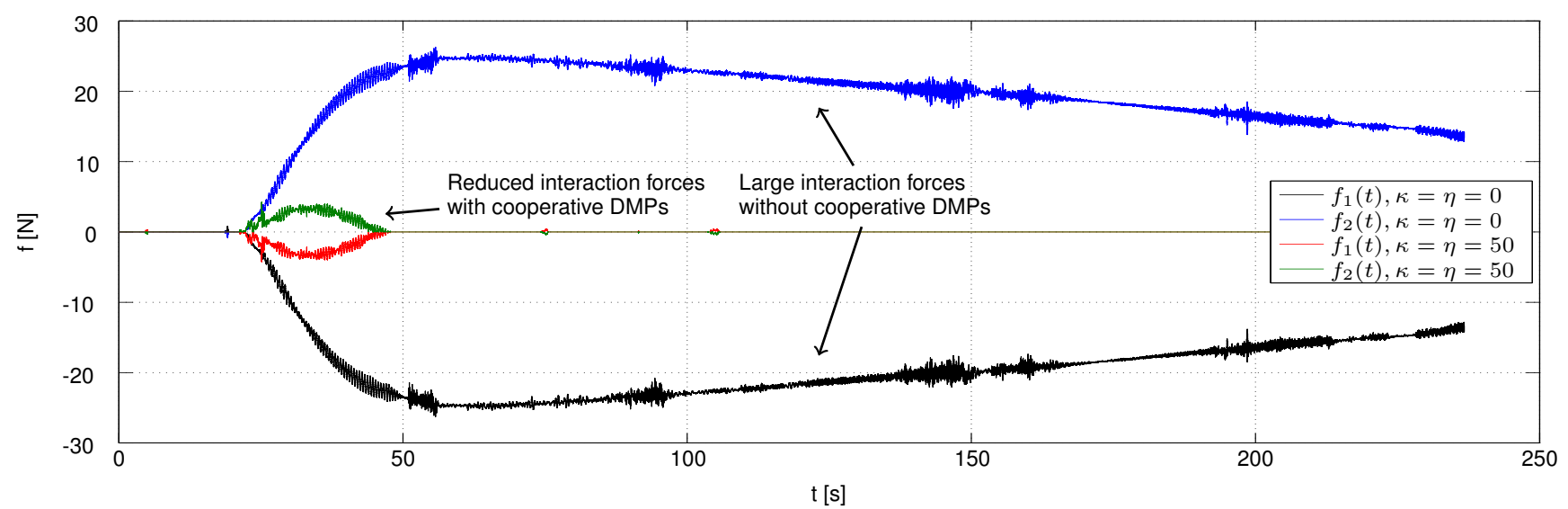

Fig. 6: Internal forces are significantly reduced during a manipulation with cooperation.

Technical Systems CoTeSys" and the European Union Seventh Framework Programme FP7/2007-2013 under grant agreement no. 601165 of the project "WEARHAP - Wearable Haptics for Humans and Robots“"

\section{REFERENCES}

[1] D. Sieber, F. Deroo, and S. Hirche, "Iterative optimal feedback controller design under relaxed rigidity constraints for cooperative manipulation," 2013, 52nd IEEE Conference on Decision and Control.

[2] J. Szewczyk, F. Plumet, and P. Bidaud, "Planning and controlling cooperating robots through distributed impedance," Journal of Robotic Systems, 2002.

[3] S. Erhart, D. Sieber, and S. Hirche, "An impedance-based control architecture for multi-robot dual-arm mobile manipulation," in Proc. IEEE/RSJ IROS, 2013.

[4] K. Kosuge, T. Oosumi, M. Satou, K. Chiba, and K. Takeo, "Transportation of a single object by two decentralized-controlled nonholonomic mobile robots," in Robotics and Automation, 1998 IEEE International Conference on, 1998.

[5] O. Khatib, K. Yokoi, K. Chang, D. Ruspini, R. Holmberg, and A. Casal, "Vehicle/arm coordination and multiple mobile manipulator decentralized cooperation," in Intelligent Robots and Systems, IEEE/RSJ International Conference on, 1996.

[6] T. G. Sugar and V. Kumar, "Control of cooperating mobile manipulators," Robotics and Automation, IEEE Transactions on, 2002.

[7] J. P. Desai and V. Kumar, "Motion planning for cooperating mobile manipulators," Journal of Robotic Systems, 1999.

[8] D. Sieber, F. Deroo, and S. Hirche, "Formation-based approach for cooperative manipulation based on iterative optimal controller design," IEEE International Conf. on Intelligent Robots and Systems, 2013.
[9] A. J. Ijspeert, J. Nakanishi, and S. Schaal, "Learning rhythmic movements by demonstration using nonlinear oscillators," in IEEE International Conference on Intelligent Robots and Systems, 2002.

[10] S. Schaal, "Dynamic movement primitives-a framework for motor control in humans and humanoid robotics," in 2nd International Symposium on Adaptive Motion of Animals and Machines, 2003.

[11] A. Gams, B. Nemec, L. Zlajpah, M. Wachter, A. Ijspeert, T. Asfour, and A. Ude, "Modulation of motor primitives using force feedback: Interaction with the environment and bimanual tasks," in Intelligent Robots and Systems, IEEE International Conference on, 2013.

[12] A. J. Ijspeert, J. Nakanishi, and S. Schaal, "Movement imitation with nonlinear dynamical systems in humanoid robots," in Robotics and Automation, IEEE International Conference on, 2002.

[13] A. Ude, A. Gams, T. Asfour, and J. Morimoto, "Task-specific generalization of discrete and periodic dynamic movement primitives,' Robotics, IEEE Transactions on, vol. 26, no. 5, pp. 800-815, 2010.

[14] S. Vijayakumar and S. Schaal, "Locally weighted projection regression: An o (n) algorithm for incremental real time learning in high dimensional space," in Proceedings of the Seventeenth International Conference on Machine Learning, 2000.

[15] S. Schaal, P. Mohajerian, and A. Ijspeert, "Dynamics systems vs. optimal control - a unifying view," Progress in brain research, 2007.

[16] H. G. Tanner, A. Jadbabaie, and G. J. Pappas, "Stable flocking of mobile agents, part i: Fixed topology," in Decision and Control, 42nd IEEE Conference on, 2003.

[17] M. Egerstedt, S. Martini, M. Cao, K. Camlibel, and A. Bicchi, "Interacting with networks: How does structure relate to controllability in single-leader, consensus networks?" Control Systems, IEEE, 2012.

[18] B. D. Anderson, C. Yu, B. Fidan, and J. M. Hendrickx, "Rigid graph control architectures for autonomous formations," Control Systems, IEEE, 2008. 